\title{
Superficial Saturation
}

\author{
Tetsuo WATANABE
}

The National Defence Academy

(Communicated by Y. Kawada).

\section{Introduction}

Let $A$ be a Cohen-Macaulay semi-local ring of dimension $d, I$ an ideal of definition of $A$ and $P(I, t)=\sum_{n>0}^{\infty} \lambda\left(I^{n} / I^{n+1}\right) t^{n}$ the associated Poincaré series where $\lambda()$ denotes the length of $A$-module. Then $P(I, t)$ is of the form $e_{0}(1-t)^{-d}-e_{1}(1-t)^{1-d}+\cdots+(-1)^{d-1} e_{d-1}(1-t)^{-1}+(-1)^{d} e_{d}^{(0)}+$ $(-1)^{d} e_{d}^{(1)} t+\cdots+(-1)^{d} e_{d}^{(r)} t^{r}$. The coefficients $e_{k}(0 \leqq k \leqq d)$ are the so called normalized Hilbert-Samuel coefficients of $I$ with $e_{d}=e_{d}^{(0)}+e_{d}^{(1)}+\cdots+e_{d}^{(r)}$. Since $\sum_{i=0}^{k}\left(\begin{array}{c}d+i-1 \\ i\end{array}\right)=\left(\begin{array}{c}k+d \\ d\end{array}\right)$, the Hilbert-Samuel function $\lambda\left(A / I^{n+1}\right)$ of $I$ equals $e_{0}\left(\begin{array}{c}n+d \\ d\end{array}\right)-e_{1}\left(\begin{array}{c}n+d-1 \\ d-1\end{array}\right)+\cdots+(-1)^{d-1} e_{d-1}\left(\begin{array}{c}n+1 \\ 1\end{array}\right)+(-1)^{d} e_{d}$ for each $n>r . \quad$ We say that $e_{0}(1-t)^{-d}+\cdots+(-1)^{d-1} e_{d-1}(1-t)^{-1}$ and $(-1)^{d}\left(e_{d}^{(0)}+\right.$ $\left.e_{d}^{(1)} t+\cdots+e_{d}^{(r)} t^{r}\right)$ are respectively the principal part and the polynomial part of the Poincaré series. In this paper we assume that $A / P$ is infinite for each maximal ideal $P$, which guarantees the existence of superficial elements. A superficial element $x$ of $I$ is said to be stable if $I^{n}: x=I^{n-1}$ for all $n>1$. We say that a sequence of $d$ elements $x_{1}, \cdots, x_{d}$ of $I$ is an $I$-superficial (resp. a stable $I$-superficial) sequence, if $x_{k} \bmod .\left(x_{1}, \cdots, x_{k-1}\right)$ is a (resp. stable) superficial element of $I /\left(x_{1}, \cdots, x_{k-1}\right)$ for each $k(1 \leqq k \leqq d)$. For an $I$-superficial sequence $x_{1}, \cdots, x_{d}$, there exists $m>0$ such that $\left(x_{1}, \cdots, x_{d}\right) I^{m}=I^{m+1}$. We evaluate $m$ in section 1 .

Now in case $d=1, I$ is said to be stable if it satisfies one of the following equivalent conditions.

(i ) $\lambda\left(A / I^{n}\right)$ is a polynomial in $n$ for all $n>0$.

(ii) $x I=I^{2}$ for some $x$ in $I$.

(iii) $P(I, t)$ is of the form $e_{0}(1-t)^{-1}-e_{1}$ (see [6]).

In the case of dimension $d>1$, the theory of stable ideals can be extended in two directions. One is about the ideals such that $\left(x_{1}, \cdots, x_{d}\right) I=I^{2}$ for some $x_{1}, \cdots, x_{d}$ in $I$. The other is about the ideals satisfying the above 
condition (i). We are mainly concerned with the latter case. So we define the stability of $I$ as such. First we define the superficial saturation of a decreasing sequence of ideals belonging to $I$. Then the sequence of ideals thus obtained has a stable superficial element and has the same associated Hilbert-Samuel polynomial. Therefore we get some information about the coefficients of the polynomial part of $P(I, t)$ by comparing them. In section 2, we show that this method is especially useful in dimension 2. As an application, we give another proof of K. Kubota's result ([5]) that, if the Hilbert-Samuel polynomial $P(n)$ equals $e_{0}\left(\begin{array}{c}n+d-1 \\ d\end{array}\right)+$ $\lambda(A / I)\left(\begin{array}{c}n+d-1 \\ d-1\end{array}\right)$, then $\lambda\left(A / I^{n+1}\right)=P(n)$ for all $n \geqq 0$.

REMARK 1. An ideal $I$ in $A$ is called open if $m^{n} \subset I$ for some $n>0$, where $m$ is the Jacobson radical of $A$. In [14] Lemma 6, we assumed implicitly that the open ideal $I$ is contained in $m$. Therefore we assume in this paper that $I$ is an ideal of definition.

REMARK 2. The definition of Cohen-Macaulay ring is that of Nagata [8]. Therefore we assume that all maximal ideals of $A$ have the same rank.

\section{§1. Superficial saturation.}

Lemma 1. Let $x_{1}, \cdots, x_{r}$ be elements of $I$. Then the following statements are equivalent.

(i) The sequence $x_{1}, \cdots, x_{r}$ is A-regular.

(ii) $A /\left(x_{1}, \cdots, x_{i-1}\right) \stackrel{x_{i}}{\rightarrow} A /\left(x_{1}, \cdots, x_{i-1}\right)$ is injective for each $i(1 \leqq i \leqq r)$.

(iii) $\left(A /\left(x_{1}, \cdots, x_{i-1}\right)\right) \otimes A_{P} \stackrel{x_{i} \otimes 1}{\longrightarrow}\left(A /\left(x_{1}, \cdots, x_{i-1}\right)\right) \otimes A_{P}$ is injective for each $i(1 \leqq i \leqq r)$ and each maximal ideal $P$ in $A$.

(iv) $A_{P} /\left(x_{1}, \cdots, x_{i-1}\right) \stackrel{x_{i}}{\rightarrow} A_{P} /\left(x_{1}, \cdots, x_{i-1}\right)$ is injective for each $i(1 \leqq i \leqq r)$ and each maximal ideal $P$ in $A$.

(v) $\operatorname{ht}_{A_{P}}\left(x_{1}, \cdots, x_{r}\right)=r$ for each maximal ideal $P$ in $A$.

Proof. (i) is equivalent to (ii) by the definition. (ii) is equivalent to (iii) by [1] Proposition 3.9. Since the functor $\otimes A_{P}$ is exact, (iii) is equivalent to (iv). (iv) is equivalent to (v) by [7] Theorem 31.

LemMA 2. Let $I$ be an ideal of definition of $A$ and $x_{1}, \cdots, x_{d}$ be elements of $I$ such that $\left(x_{1}, \cdots, x_{d}\right) I^{m}=I^{m+1}$ for some $m>0$. Then the sequence $x_{1}, \cdots, x_{d}$ is A-regular.

Proof. Let $P$ be a maximal ideal of $A$. Then we have $d=\mathrm{ht}_{A_{P}}\left(I A_{P}\right)=$ 
$\mathrm{ht}_{A_{P}}\left(I^{m+1} A_{P}\right) \leqq \mathrm{ht}_{A_{P}}\left(x_{1}, \cdots, x_{d}\right) \leqq \mathrm{ht}_{A_{P}}\left(I A_{P}\right)=d$. Hence $\mathrm{ht}_{A_{P}}\left(x_{1}, \cdots, x_{d}\right)=d$. By Lemma $1, x_{1}, \cdots, x_{d}$ is an $A$-regular sequence.

LEMma 3. Let $x_{1}$ be a superficial element of $I$. Then there exists elements $x_{2}, \cdots, x_{d}$ of $I$ such that $x_{1}, \cdots, x_{d}$ is an I-superficial sequence and $\left(x_{1}, \cdots, x_{d}\right) I^{m}=I^{m+1}$ for some $m>0$.

Proof. By induction on $d$, the proof proceeds just as the one given in [14] Lemma 6.

Lemma 4. Let $x_{1}, \cdots, x_{d}$ be an A-regular sequence in $I$. If $a$ is in $\left(x_{1}, \cdots, x_{d}\right)^{r} I^{m}: x_{1}$, then $a$ is a homogeneous polynomial of degree $r-1$ in $I\left[x_{1}, \cdots, x_{d}\right]$.

Proof. Using the condition (*) of [7] (15.B) repeatedly, we know that $a$ is a homogeneous polynomial of degree $r-1$ in $I\left[x_{1}, \cdots, x_{d}\right]$.

LEMMA 5. If $\left(x_{1}, \cdots, x_{d}\right) I=I^{2}$ for some $x_{1}, \cdots, x_{d}$ in $I$, then $x_{1}, \cdots, x_{d}$ is a stable I-superficial sequence.

Proof. Since $x_{1}, \cdots, x_{d}$ is $A$-regular by Lemma 2 , the lemma follows from Lemma 4.

LEMMA 6. Let $x_{1}$ be a superficial element of $I$. Then there is an integer $s>0$ such that $I^{n}: x_{1}=I^{n-1}$ for each $n>s$.

Proof. Let $r>0$ be an integer such that $\left(I^{n}: x_{1}\right) \cap I^{r}=I^{n-1}$ for each $n>r$ and $x_{1}, \cdots, x_{d}$ be an $I$-superficial sequence such that $\left(x_{1}, \cdots, x_{d}\right) I^{m}=$ $I^{m+1}$ for some $m>0$. Put $s=m+r$. Then, for each $n>s$ and each $a$ in $I^{n}: x_{1}$, we have $a x_{1} \in I^{n}=\left(x_{1}, \cdots, x_{d}\right)^{r} I^{n-r}$. By Lemma $4, a$ is in $I^{r}$. Thus $a$ is in $I^{n-1}$.

Proposition 7. Let $x$ be a superficial element and $s$ the least integer $s>0$ such that $I^{n}: x=I^{n-1}$ for each $n>s$. Then $s$ is independent of the choice of the superficial element $x$.

Proof. Let $x, y$ be superficial elements of $I$ and $s(x)$ and $s(y)$ the least such integers respectively for $x$ and $y$. Let $n>s(x)$. Then, for any $z$ in $I^{n}: y$, we have $y z x^{s(y)} \in I^{n+s(y)}$. Hence $z x^{s(y)} \in I^{n+s(y)-1}$. Thus $z \in I^{n-1}$. Therefore $I^{n}: y=I^{n-1}$, which means $s(x) \geqq s(y)$. By the change of the role of $x$ and $y$, we get $s(x)=s(y)$.

We denote this $s$ by $s(I)$.

COROLlaRy 8. If I has a stable superficial element, then any superficial element of $I$ is stable. 
Let $m(I)$ be the least integer $m \geqq 0$ such that $\lambda\left(A / I^{n}\right)$ is a polynomial in $n$ for each $n>m$. If $m(I)>0$, then $m(I)$ is the degree of the polynomial part of the Poincare series $P(I, t)$. We say that $I$ is stable if $m(I)=0$, in other words, if the polynomial part of the Poincaré series $P(I, t)$ is a constant.

Proposition 9. Let $x$ be a superficial element of $I, \bar{A}=A /(x)$ and $\bar{I}=$ $I /(x)$. Then $s(I) \leqq \max \{m(I)+1, m(\bar{I})\}$. If $m(I)+1<m(\bar{I})$, then $s(I)=m(\bar{I})$.

Proof. Let $N_{n}=x A \cap I^{n}$. Then we have the exact sequence $0 \rightarrow$ $x A / N_{n} \rightarrow A / I^{n} \rightarrow \bar{A} / \bar{I}^{n} \rightarrow 0$. Hence

$$
\lambda\left(A / I^{n}\right)=\lambda\left(x A / N_{n}\right)+\lambda\left(\bar{A} / \bar{I}^{n}\right) .
$$

Let $m(N)$ be the least integer $m \geqq 0$ such that $\lambda\left(x A / N_{n}\right)$ is a polynomial in $n$ for each $n>m$. (i) Assume that $m(I)<m(\bar{I})$. Then we know that $m(N)=m(\bar{I})$ by $(*)$. Since $\lambda\left(x A / N_{n}\right)=\lambda\left(x A+I^{n} / I^{n}\right)=\lambda\left(A /\left(I^{n}: x\right)\right)=\lambda\left(A / I^{n-1}\right)$ for each $n>s(I)$ and both $\lambda\left(x A / N_{n}\right)$ and $\lambda\left(A / I^{n-1}\right)$ are polynomials in $n$ for each $n>m(\bar{I}), \lambda\left(x A / N_{n}\right)=\lambda\left(A / I^{n-1}\right)$ for each $n>m(\bar{I})$. Hence $I^{n}: x=I^{n-1}$ for each $n>m(\bar{I})$. Thus $s(I) \leqq m(N)=m(\bar{I})$. (ii) Assume that $m(\bar{I}) \leqq m(I)$. Then from (*), $m(N) \leqq m(I)$. Since $\lambda\left(A / N_{n}\right)=\lambda\left(A /\left(I^{n}: x\right)\right)=\lambda\left(A / I^{n-1}\right)$ for each $n>m(I)+1$, we have $I^{n}: x=I^{n-1}$. Thus $s(I) \leqq m(I)+1$. Finally suppose $m(I)+1<m(\bar{I})$. Then $m(N)=m(\bar{I})$ by $(*)$. Assume that $s(I)<m(N)$. Since $m(N)-1 \geqq s(I)$, we have $I^{n}: x=I^{n-1}$ for each $n>m(N)-1$. Hence $\lambda\left(x A / N_{n}\right)=\lambda\left(A / I^{n-1}\right)$ is a polynomial in $n$ for each $n>m(N)-1$ because $n-1>m(N)-2 \geqq m(I)$, a contradiction.

Proposition 10. Let $x_{1}, \cdots, x_{d}$ be an I-superficial sequence, $I_{i}=$ $I /\left(x_{1}, \cdots, x_{i}\right)(1 \leqq i \leqq d-1)$ and $m=1+\max \left\{m(I), m\left(I_{1}\right), \cdots, m\left(I_{d-1}\right)\right\}$. Then $\left(x_{1}, \cdots, x_{d}\right) I^{m}=I^{m+1}$.

Proof. If $d=1$, the proposition follows from [6] Theorem 1.9. Let $d>1$ and assume the proposition for $d-1$. Then $\left(x_{1}, \cdots, x_{d}\right) I^{m} \equiv$ $I^{m+1} \bmod . x_{1} A$. Hence $x_{1} A+\left(x_{2}, \cdots, x_{d}\right) I^{m} \supset I^{m+1}$. Since $m \geqq s(I)$, we deduce that $\left(x_{1}, x_{2}, \cdots, x_{d}\right) I^{m}=I^{m+1}$.

Proposition 11. Under the same assumptions as in Proposition 10, the following statements are equivalent.

(i) $I, I_{1}, \cdots, I_{d-1}$ are stable.

(ii) $P(I, t)=e_{0}(1-t)^{-d}-e_{1}(1-t)^{1-d}$.

(iii) $\lambda\left(A / I^{n+1}\right)=e_{0}\left(\begin{array}{c}n+d \\ d\end{array}\right)-e_{1}\left(\begin{array}{c}n+d-1 \\ d-1\end{array}\right)$ for all $n \geqq 0$.

(iv) $\lambda\left(A / I^{n+1}\right)=e_{0}\left(\begin{array}{c}n+d \\ d\end{array}\right)-e_{1}\left(\begin{array}{c}n+d-1 \\ d-1\end{array}\right)$ for all large $n$ and $e_{d}^{(0)}=0$. 
( v ) $\left(x_{1}, \cdots, x_{d}\right) I=I^{2}$.

Proof. Assume (i). If $d=1$, (i) implies (ii) by [6] Theorem 1.9. Let $d>1$. By Proposition 9, there exists a stable $I$-superficial sequence $x_{1}, \cdots, x_{d}$. Since $\lambda\left(x_{1} A / N_{n}\right)=\lambda\left(A / I^{n-1}\right)$, the equality $(*)$ in the proof of Proposition 9 implies that $P(I, t)=P\left(I_{1}, t\right) /(1-t)$. Now (ii) follows by induction on $d$. Obviously (ii) is equivalent to (iii) and (iii) implies (iv). (v) follows from (iv) by [5] Corollary 6 and Proposition 8. Assume (v). By Lemma $5, x_{1}, \cdots, x_{d}$ is a stable $I$-superficial sequence. Thus $P\left(I_{i-1}, t\right)=$ $P\left(I_{i}, t\right) /(1-t) \quad(1 \leqq i \leqq d-1)$. Since $P\left(I_{d-1}, t\right)=e_{0}(1-t)^{-1}-e_{1}, \quad P\left(I_{i}, t\right)=$ $e_{0}(1-t)^{i-d}-e_{1}(1-t)^{i+1-d}$. Therefore $I_{i}$ is stable $(0 \leqq i \leqq d-1)$.

REmark. The conditions (i) and (v) of Proposition 11 are independent of the choice of the $I$-superficial sequence $x_{1}, \cdots, x_{d}$, because the conditions (ii), (iii) and (iv) are so.

Definition. We say that a family of ideals of definition of $A$, $\left\{J^{(n)}\right\}_{n>0}$ is a decreasing sequence belonging to $I$ if it satisfies the following conditions.

(i) $J^{(n)} \supset J^{(n+1)}$ for each $n>0$.

(ii) $J^{(n)} J^{(m)} \subset J^{(n+m)}$ for each $n, m>0$.

(iii) $I^{n} \subset J^{(n)}$ for each $n>0$ and $I^{n}=J^{(n)}$ for all large $n$.

Let $\left\{J^{(n)}\right\}_{n>0}$ be a decreasing sequence belonging to $I$. Then we say that $P(t)=\sum_{n \geq 0} \lambda\left(J^{(n)} / J^{(n+1)}\right) t^{n}\left(J^{(0)}=A\right)$ and $H(n)=\lambda\left(A / J^{(n+1)}\right)$ are respectively the Poincare series and the Hilbert-Samuel function of $\left\{J^{(n)}\right\}_{n>0}$. By the condition (iii), $I$ and $\left\{J^{(n)}\right\}_{n>0}$ have the same Hilbert-Samuel polynomial. Therefore the principal parts of their Poincaré series are the same.

LEMMA 12. If $x$ is a superficial element of $I$, then $x$ is a superficial element of $I A_{P}$ for each maximal ideal $P$ in $A$ and $s\left(I A_{P}\right) \leqq s(I)$.

Proof. Since $0 \rightarrow I^{m-1} \rightarrow A \stackrel{x}{\rightarrow} A / I^{m}$ is exact for each $m>s(I)$ by Lemma 6 , we have the exact sequence; $0 \rightarrow I^{m-1} A_{P} \rightarrow A_{P} \stackrel{x}{\rightarrow} A_{P} / I^{m} A_{P}$ for each $m>s(I)$. Thus $x$ is a superficial element of $I A_{P}$ and $s\left(I A_{P}\right) \leqq s(I)$.

Let $x$ be a superficial element of $I$. Then the least integer $s\left(J^{(*)}\right)>0$ such that $J^{(n)}: x=J^{(n-1)}$ for each $n>s\left(J^{(*)}\right)$ is independent of the choice of $x$. If $s\left(J^{(*)}\right)=1$, we say that $x$ is a stable superficial element of the decreasing sequence $\left\{J^{(n)}\right\}_{n>0}$. Put $I^{(n)}=\bigcup_{k>0}\left(J^{(n+k)}: x^{k}\right)$. As $\left(J^{(n+k)}: x^{k}\right) \subset$ $\left(J^{(n+k+1)}: x^{k+1}\right)$ for each $k>0$ and $A$ is Noetherian, $I^{(n)}=I^{n+k}: x^{k}$ for all large $k$. Obviously $J^{(n)} \subset I^{(n)}$.

LEMMA 13. $I^{(n)}$ is independent of the choice of the superficial 
element $x$.

Proof. Let $y$ be a superficial element of $I$ and $m>s(I)$. Then $a x^{m} \in I^{n+m}$ if and only if $a x^{m} y^{m} \in I^{n+2 m}$. Hence we have $I^{n+m}: x^{m}=I^{n+m}: y^{m}$.

LEMMA 14. $I^{(n)}$ is contained in the Jacobson radical of $A$ for each $n>0$.

Proof. Assume the contrary. Then there exists $a \in I^{(n)}$ for some $n>0$ which is not contained in some maximal ideal $P$ in $A$. Since $a x^{m} \in I^{n+m}$ for some large $m>0, a x^{m} \in I^{n+m} A_{P}$. As $a$ is not in $P, a$ is a unit in $A_{P}$. Hence $x^{m} \in I^{n+m} A_{P}$. Let $k$ be an integer such that $k \geqq s(I)$ and $k>n$. For any element $b$ of $I^{k-n} A_{P}$, we have $b x^{m} \in I^{k+m} A_{P}$. By Lemma 12, $b \in I^{k} A_{P}$. Hence $I^{k-n} A_{P} \subset I^{k} A_{P}$. Therefore $I^{k-n} A_{P}=I^{k} A_{P}$. Вy Nakayama's lemma, $I^{k-n} A_{P}=0$, a contradiction.

Definition. We say that the decreasing sequence $\left\{I^{(n)}\right\}_{n>0}$ thus obtained is the superficial saturation of $\left\{J^{(n)}\right\}_{n>0}$. Remark that the superficial saturation is uniquely determined by $I$. We say that $I$ is superficially saturated if $I^{(1)}=I$. A decreasing sequence $\left\{J^{(n)}\right\}_{n>0}$ is said to be superficially saturated if $J^{(n)}=I^{(n)}$ for each $n>0$.

Proposition 15. Let $x$ be a superficial element of $I,\left\{J^{(n)}\right\}_{n>0} a$ decreasing sequence belonging to $I$ and $\left\{I^{(n)}\right\}_{n>0}$ the superficial saturation of $\left\{J^{(n)}\right\}_{n>0}$. Then

(i) $\left\{I^{(n)}\right\}_{n>0}$ is a decreasing sequence belonging to $I$.

(ii) $\left\{I^{(n)}\right\}_{n>0}$ has a stable superficial element.

(iii) $\left\{I^{(n)}\right\}_{n>0}$ is superficially saturated.

Proof. (i) By Lemma 14, $I^{(n)}$ is an ideal of definition of $A$. Obviously $I^{(n)} \supset I^{(n+1)}$ for each $n>0$. Let $a \in I^{(n)}$ and $b \in I^{(m)}$. Then $a x^{k} \in I^{n+k}$ and $b x^{k} \in I^{m+k}$ for some $k$. Hence $a b x^{2 k} \in I^{n+m+2 k}$. Thus $a b \in I^{(n+m)}$. Obviously $I^{n} \subset I^{(n)}$ for each $n>0$. Let $m$ be an integer such that $I^{(n)}=I^{n+m}: x^{m}$. Then, if $n \geqq s(I)$, we have $I^{(n)}=I^{n}$. Thus $\left\{I^{(n)}\right\}_{n>0}$ is a decreasing sequence belonging to $I$. (ii) Let $k>1$ and $a \in I^{(k)}: x$. Then $a x \in I^{(k)}$. Hence $a x^{m+1} \in I^{k+m}$ for some $m$. This implies that $a \in I^{(k-1)}$. (iii) follows from (ii).

COROLlaRY 16. I has a stable superficial element if and only if $I^{(n)}=I^{n}$ for each $n>0$.

Lemma 17 (One dimensional case). Assume that $A$ is of dimension one. Let $x$ be a regular element of $A$ contained in $I, e_{0}$ the multiplicity of $I$ and $\left\{J^{(n)}\right\}_{n>0}$ a decreasing sequence belonging to $I$. Then $e_{0}=\lambda(A / x A) \geqq$ 
$\lambda\left(J^{(n)} / J^{(n+1)}\right)$ for each $n \geqq 0$. The equality holds if and only if $x J^{(n)}=J^{(n+1)}$. If $x J^{(n)}=J^{(n+1)}$, then $x J^{(m)}=J^{(m+1)}$ for each $m \geqq n$.

Proof. $e_{0}=\lambda(A / x A)$ by [6] Theorem 1.9. Since multiplication by $x$ induces the isomorphism $A / J^{(n)} \rightarrow x A / x J^{(n)}$, we have

$$
\begin{aligned}
\lambda(A / x A) & =\lambda(A / x A)+\lambda\left(x A / x J^{(n)}\right)-\lambda\left(A / J^{(n)}\right) \\
& =\lambda\left(A / x J^{(n)}\right)-\lambda\left(A / J^{(n)}\right) \\
& =\lambda\left(J^{(n)} / x J^{(n)}\right) \geqq \lambda\left(J^{(n)} / J^{(n+1)}\right) .
\end{aligned}
$$

From this, it is clear that the equality holds if and only if $x J^{(n)}=J^{(n+1)}$. Assume that $x J^{(n)}=J^{(n+1)}$. It is obvious that $x J^{(m)} \subset J^{(m+1)}$ for each $m \geqq 0$. Let $m \geqq n$ and $y \in J^{(m+1)}$. Then $y x^{k} \in J^{(m+1+k)}$ for some $k$. As $y \in J^{(n+1)}$, $y=x z$ for some $z \in J^{(n)}$. Thus $z x^{k+1} \in J^{(m+1+k)}$. This implies that $z \in J^{(m)}$.

CoROLlaRy 18. Let $A$ be of dimension one and $\left\{J^{(n)}\right\}_{n>0}$ a decreasing sequence belonging to $I$. Then all the normalized coefficients of the polynomial part of the Poincaré series of $\left\{J^{(n)}\right\}_{n>0}$ are non-negative.

LEMma 19. Let $x$ be a superficial element of $I,\left\{I^{(n)}\right\}_{n>0}$ the superficial saturation of $\left\{I^{n}\right\}_{n>0}$ and $(-1)^{d}\left(a_{0}+a_{1} t+\cdots+a_{r} t^{r}\right)$ the polynomial part of the Poincaré series of $\left\{I^{(n)}\right\}_{n>0}$. Then $e_{0}\left(I^{(*)}\right)=e_{0}\left(I^{(*)} /(x)\right), \cdots, e_{d-2}\left(I^{(*)}\right)=$ $e_{d-2}\left(I^{(*)} /(x)\right)$ and the polynomial part of $\left\{I^{(n)}+(x) /(x)\right\}_{n>0}$ is of the form $(-1)^{d-1}\left(b_{0}+b_{1} t+\cdots+b_{r+1} t^{r+1}\right)$, where $e_{d-1}=b_{0}+\cdots+b_{r+1}, a_{0}=b_{1}+b_{2}+\cdots+b_{r+1}$, $a_{1}=b_{2}+b_{3}+\cdots+b_{r+1}, \cdots, a_{r-1}=b_{r}+b_{r+1}, a_{r}=b_{r+1}$.

Proof. Let $N_{n}=x A \cap I^{(n)}$. Then, from the exact sequence $0 \rightarrow x A / N_{n} \rightarrow$ $A / I^{(n)} \rightarrow \bar{A} / \bar{I}^{(n)} \rightarrow 0$, we have $(1-t) P\left(I^{(*)}, t\right)=P\left(\bar{I}^{(*)}, t\right)$, just as the proof of Proposition 9. Comparing the coefficients, we have the lemma.

Proposition 20. If there exists a stable I-superficial sequence, then all the normalized coefficients $e_{i}(1 \leqq i \leqq d)$ of the Hilbert-Samuel polynomial of $I$ and all the normalized coefficients $e_{d}^{(i)}(0 \leqq i \leqq m(I))$ of the polynomial part of the Poincaré series of I are non-negative.

Proof. This follows from Lemma 19 by using induction on the dimension of $A$.

LEMMA 21. Let $\left\{J^{(n)}\right\}_{n>0}$ be a decreasing sequence belonging to $I$ and let $a_{0}+a_{1} t+\cdots+a_{m} t^{m}, b_{0}+b_{1} t+\cdots+b_{l} t^{l}$ and $a_{0}^{*}+a_{1}^{*} t+\cdots+a_{n}^{*} t^{n}$ be respectively polynomial parts of the Poincaré series of $I,\left\{J^{(n)}\right\}_{n>0}$ and $\left\{I^{(n)}\right\}_{n>0}$. Then $a_{0}+a_{1}+\cdots+a_{k} \geqq b_{0}+b_{1}+\cdots+b_{k} \geqq a_{0}^{*}+a_{1}^{*}+\cdots+a_{k}^{*}$ for each $k \geqq 0$.

Proof. Since the Poincaré series of these decreasing sequence have 
the same principal part, the inequality $\lambda\left(A / I^{k+1}\right) \geqq \lambda\left(A / J^{(k+1)}\right) \geqq \lambda\left(A / I^{(k+1)}\right)$ implies the lemma.

\section{§2. Two dimensional case.}

THEOREM 22. Let $A$ be of dimension 2 and $a_{0}^{*}+a_{1}^{*} t+\cdots+a_{m}^{*} t^{m}\left(a_{m}^{*} \neq 0\right)$ the polynomial part of the Poincaré series of a superficially saturated decreasing sequence $\left\{I^{(n)}\right\}_{n>0}$ belonging to $I$. Then $a_{0}^{*}>a_{1}^{*}>\cdots>a_{m}^{*}>0$.

Proof. Let $x$ be a superficial element of $I$. Then $P\left(I^{(*)}, t\right)=$ $P\left(I^{(*)} /(x), t\right) /(1-t)$. Now the theorem follows from Lemma 17 and Lemma 19.

COROLlaRY 23. Let $A$ be of dimension $d \geqq 2$. Then $e_{2}(I) \geqq 0$.

Proof. Since the Hilbert-Samuel polynomials of $\left\{I^{(n)}\right\}_{n>0}$ and $\left\{I^{n}\right\}_{n>0}$ are the same, we may consider $\left\{I^{(n)}\right\}_{n>0}$ instead of $\left\{I^{n}\right\}_{n>0}$. By Lemma 19, it is sufficient to prove the lemma in the case of dimension 2. Now the lemma follows from Theorem 22.

Theorem 24. Let $A$ be of dimension 2 and let $I$ be stable and superficially saturated. Then $\left\{I^{n}\right\}_{n>0}$ is the only decreasing sequence belonging to $I$.

Proof. With the same notations as in Lemma 21, we have $a_{0}=b_{0}=a_{0}^{*}$ and $a_{1}+\cdots+a_{k} \geqq b_{1}+\cdots+b_{k} \geqq a_{1}^{*}+\cdots+a_{k}^{*}$ for each $k \geqq 1$. Since $a_{k}=0$ and $a_{k}^{*} \geqq 0$ for each $k \geqq 1$, we have $b_{k}=0$ for each $k \geqq 1$.

CoROLlary 25. Let $A$ be of dimension 2 and let I be stable and superficially saturated. Then there exists a stable superficial element of $I$.

Proof. By Theorem 24, $I^{(k)}=I^{k}$ for each $k \geqq 1$.

Let $m\left(J^{(*)}\right)$ be the least non-negative integer such that $J^{(n)}: x=J^{(n-1)}$ for each $n>m\left(J^{(*)}\right)$, where $x$ is a superficial element of $I$ and $\left\{J^{(n)}\right\}_{n>0}$ is a decreasing sequence belonging to $I$.

Proposition 26. Let $\left\{I^{(n)}\right\}_{n>0}$ be the superficial saturation of a decreasing sequence $\left\{J^{(n)}\right\}_{n>0}$ belonging to $I, m=m\left(J^{(*)}\right)$ and $n=m\left(I^{(*)}\right)$. Then:

(i) If $A$ is of dimension 1, then $x J^{(m+1)}=J^{(m+2)}$ for each superficial element $x$.

(ii) If $A$ is of dimension 2, then $(x, y) I^{(n+2)}=I^{(n+8)}$ for each I-superficial sequence $x, y$. In particular, if $I$ is stable and superficially saturated, then $(x, y) I^{2}=I^{3}$. 
Proof. (i) follows from Lemma 17. (ii) Let $x, y$ be an $I$-superficial sequence. Then $m\left(I^{(*)} /(x)\right)=m+1$. Hence $y I^{(m+2)} /(x)=I^{(m+3)} /(x)$ by (i). As $x$ is a stable superficial element of $\left\{I^{(n)}\right\}_{n>0}$, we have $(x, y) I^{(m+2)}=I^{(m+8)}$.

Proposition 27. Let $A$ be of dimension 2 and $k$ a positive integer. Then the decreasing sequence $\left\{I^{(n k)}\right\}_{n>0}$ belonging to $I^{k}$ is stable if and only if $k>m\left(I^{(*)}\right)$.

Proof. Let $a_{0}+a t_{1}+\cdots+a_{m} t^{m}\left(a_{m} \neq 0\right)$ be the polynomial part of the Poincaré series of $\left\{I^{(n)}\right\}_{n>0}$. Then the polynomial part of the Poincaré series of $\left\{I^{(n k)}\right\}_{n>0}$ is

$$
\left(a_{0}+\cdots+a_{k-1}\right)+\left(a_{k}+\cdots+a_{2 k-1}\right) t+\cdots .
$$

Now the proposition follows from Theorem 22.

\section{§3. An application.}

As an application of superficial saturation, we prove the implication (iv) $\Rightarrow$ (v) of Proposition 11 .

THEOREM 28 (K. Kubota). Let $\lambda(A / I)=e_{0}-e_{1}$ and $\lambda\left(A / I^{n+1}\right)=e_{0}\left(\begin{array}{c}n+d \\ d\end{array}\right)-$ $e_{1}\left(\begin{array}{c}n+d-1 \\ d-1\end{array}\right)$ for all large $n$. Then $\left(x_{1}, \cdots, x_{d}\right) I=I^{2}$ for each I-superficial sequence $x_{1}, \cdots, x_{d}$.

Proof. Let $x_{1}$ be a superficial element of $I, \bar{I}=I /\left(x_{1}\right),\left\{I^{(n)}\right\}_{n>0}$ the superficials aturation of $\left\{I^{n}\right\}_{n>0}$ and $\bar{I}^{(n)}=I^{(n)}+\left(x_{1}\right) /\left(x_{1}\right)$. If $d=1$, then the theorem follows from [6] Theorem 1.9. Assume that $d=2$. Since $e_{d}^{(0)}(I)=0$, we have $0=e_{d}^{(0)}\left(I^{(*)}\right)=e_{d}^{(1)}\left(I^{(*)}\right)=e_{d}^{(2)}\left(I^{(*)}\right)=\cdots$ by Theorem 22. As $e_{d}^{(0)}\left(I^{(*)}\right)=$ $e_{d}^{(0)}(I)$ and the principal parts of the Poincaré series of $\left\{I^{(n)}\right\}_{n>0}$ and $I$ are the same, we have $I^{(1)}=I$. By Lemma $19, m\left(\bar{I}^{(*)}\right)=0$. By Proposition 26 (i), $x_{2} \bar{I}^{(1)}=\bar{I}^{(2)}$ for some $x_{2} \in I$. Since $\bar{I}^{(2)} \supset \bar{I}^{2}$ and $x_{2} \bar{I}^{(1)}=x_{2} \bar{I} \subset \bar{I}^{2}, x_{2} \bar{I}=\bar{I}^{2}$, i.e. $x_{2} I \equiv I^{2}$ mod. $\left(x_{1}\right)$. As $I$ is superficially saturated, we have $\left(x_{1}, x_{2}\right) I=I^{2}$. Now assume that $d>2$ and we proceed by induction on $d$. By Lemma 19, the Poincaré series of $\left\{\bar{I}^{(n)}\right\}_{n>0}$ is of the form

$$
e_{0}(1-t)^{1-d}-e_{1}(1-t)^{2-d}+\cdots+(-1)^{d-1}\left(b_{0}+b_{1} t+\cdots+b_{r} t^{r}\right),
$$

where $b_{0}+b_{1}+\cdots+b_{r}=e_{d-1}=0$. Since $\left\{\bar{I}^{(n)}\right\}_{n>0}$ and $\bar{I}$ have the same HilbertSamuel polynomial, $e_{d-1}(\bar{I})=b_{0}+b_{1}+\cdots+b_{r}=0$. As $e_{0}-e_{1}=\lambda(A / I)=\lambda(\bar{A} / \bar{I})$, we can apply the induction assumption to $\bar{I}$ to know that $\left(\bar{x}_{2}, \cdots, \bar{x}_{d}\right) \bar{I}=\bar{I}^{2}$ for some $\bar{I}$-superficial sequence $\bar{x}_{2}, \cdots, \bar{x}_{d}$. By Lemma $5, \bar{x}_{2}$ is a stable superficial element of $\bar{I}$. Therefore each decreasing sequence belonging 
to $\bar{I}$ coincides with $\left\{\bar{I}^{n}\right\}_{n>0}$ by Lemma 21. Thus $\bar{I}^{(n)}=\bar{I}^{n}$ for each $n>0$. As $e_{0}-e_{1}=\lambda(\bar{A} / \bar{I})=\lambda\left(\bar{A} / \bar{I}^{(1)}\right)$, we have $b_{0}=0$. Therefore $e_{d}^{(0)}\left(I^{(*)}\right)=b_{1}+b_{2}+$ $\cdots+b_{r}=0$. Since $e_{d}^{(0)}(I)=0$, we know tha $I^{(1)}=I$, namely $I$ is superficially saturated, because the principal parts of the Poincare series of $\left\{I^{(n)}\right\}_{n>0}$ and $I$ are the same. As $\left(x_{2}, \cdots, x_{d}\right) I \equiv I^{2} \bmod .\left(x_{1}\right)$ and $I$ is superficially saturated, we have $\left(x_{1}, \cdots, x_{d}\right) I=I^{2}$.

\section{References}

[1] M. F. AtiYah and I. G. Macdonald, Introduction to Commutative Algebra, AddisonWesley, Reading, Mass., 1969.

[2] J.P. FillmoRe, On the coefficients of the Hilbert-Samuel polynomial, Math. Z., 97 (1967), 212-228.

[ 3 ] D. KIRBY, A Note on Superficial Elements of an ideal in a local ring, Quart. J. Math. Oxford Ser. (2), 14 (1963), 21-28.

[4] K. КивотA, On the Hilbert-Samuel function, Tokyo J. Math., 8 (1985), 437-448.

[5] K. KuвотA, On the stable ideals, Tokyo J. Math., 8 (1985), 449-454.

[6] J. Lipman, Stable ideals and arf Rings, Amer. J. Math., 93 (1971), 649-685.

[7] H. Matsumura, Commutative Algebra, 2nd Ed., Benjamin, New York, 1980.

[8] M. NAGATA, Local Rings, Interscience, New York, 1963.

[9] M. NARITA, A Note on the coefficients of Hilbert characteristic functions in semi-regular local rings, Proc. Cambridge Philos. Soc., 59 (1963), 269-275.

[10] D. G. NorthcotT, A Note on the coefficients of the abstract Hilbert function, J. London Math. Soc., 35 (1960), 208-214.

[11] J.D. SALLY, Cohen-Macaulay local rings of maximal embedding dimension, J. Algebra, 56 (1979), 168-183.

[12] J. D. SALLY, Super-regular sequences, Pacific J. Math., 84 (1979), 465-481.

[13] C. P. L. Rhodes, The Hilbert-Samuel polynomial in a filtered module, J. London Math. Soc. (2), 3 (1971), 73-85.

[14] T. Watanabe, On the Transversal Elements of an Open Ideals, Comment. Math. Univ. St. Paul., 31 (1982), 213-218.

Present Address:

Department of Mathematics

The National Defence Academy

YokosuKa 239 